In: In N. Gold (Ed.) (2005), Teamwork: Multi-disciplinary perspectives (pp. 216-235). Basingstoke: Palgrave Macmillan.

\title{
Evolution of Cooperation Without Awareness in Minimal Social Situations
}

\section{Andrew M. Colman}

A surprising prediction from a simple evolutionary game-theoretic model, based on meagre assumptions, is that a form of cooperation can evolve among agents acting without any deliberate intention to cooperate. There are circumstances in which agents can learn to behave cooperatively without even becoming aware of their strategic interdependence. This phenomenon occurs in what are called minimal social situations, through an unconscious mechanism of adaptive learning in pairs or groups of agents lacking any deliberate intention to cooperate. ${ }^{1}$ Whether or not it is reasonable to interpret this as a form of teamwork is debatable. The argument pivots on what are considered to be the essential or prototypic features of teamwork, and other contributors to this volume are better qualified than I to analyse this conceptual issue. But what seems uncontroversial is that minimal social situations represent special or limiting cases that should interest people who study teamwork and may help to throw light on more complex forms of teamwork. Minimal social situations are curiosities, outside the mainstream of evolutionary game theory, but the underlying theory is intrinsically interesting and may turn out to have some utility in explaining the evolution of social behaviour in conditions of incomplete information.

The increasing popularity of evolutionary games, from the closing decades of the second millennium onwards, has been fuelled by a growing suspicion that orthodox game theory, based on ideally rational players, may be irredeemably indeterminate. Orthodox game theory seeks to specify the strategies that would be chosen by rational players - rational in the sense of invariably acting to maximize their expected utilities relative to their knowledge and beliefs. It is easy to prove, via the celebrated Indirect Argument ${ }^{2}$ of von Neumann and Morgenstern (1944, pp. 146-8), that if a game has a uniquely rational solution, then that solution necessarily comprises a profile of strategies that we now call a Nash equilibrium in which each strategy is a utility-maximizing best reply to the combined strategies of the remaining players. But most interesting games, apart from those that are strictly competitive (finite, two-person, zero-sum), have multiple Nash equilibria that are neither equivalent nor interchangeable, and this implies that rational players, having identified the equilibria of a game, are left with a problem of equilibrium selection. It is in this sense that classical game theory, based on Nash equilibrium, is indeterminate.

Evolutionary game theory came into its own following the partial eclipse of the purely rational and normative approach set out by the founding game theorists. ${ }^{3}$ It deals with nonrational strategic interaction driven by mindless adaptive processes based on trial and error. It can be traced to a passage in John Nash's doctoral thesis, completed in 1950 but not published in full until more than half a century later (Nash 2002). Evolutionary game models are designed to explore the behaviour of goal-directed automata in simulated strategic interactions (Hofbauer and Sigmund, 1998; Weibull, 1995). In some models, based on adaptive learning mechanisms, individual automata adjust their strategy choices in response to the payoffs they receive in simulated interactions; in others, based on replicator dynamics, the relative proportions of different types of automata in the population change in response to payoffs. In either case, the interacting automata are programmed to maximize their individual payoffs, even though their strategy choices are made without conscious thought or deliberate choice. Evolutionary models generally converge to the vicinity of evolutionarily stable strategies, ${ }^{4}$ which are invulnerable to evolutionary invasion by alternative strategies and (it 
turns out) are invariably Nash equilibria, and this process can mimic rational interactive choice analogously to the way in which biological evolution often mimics intelligent design. Minimal social situations provide vivid examples of this.

In the paragraphs that follow, I shall explain the fundamental ideas behind two-person and multi-person minimal social situations and provide some intuitive background to the evolution of cooperation without awareness. I shall then outline a formal theory designed to explain these process in both dyads and larger groups and review some admittedly sparse empirical evidence from experiments with human decision makers in artificially contrived minimal social situations. Finally, I shall attempt to draw the threads together and discuss the implications of cooperation without awareness. 5

\section{Two-person minimal social situation}

The minimal social situation (MSS) was first described by the US psychologist Joseph Sidowski in his doctoral dissertation and in articles based on it (Sidowski, 1957; Sidowski, Wyckoff and Tabory, 1956). The two-person or dyadic MSS is a game of incomplete information in which both players know their own strategy sets but neither knows the coplayer's strategy set nor either player's payoff function. In its most extreme form, both players suffer from a profound and debilitating kind of ignorance: they are oblivious not only of the nature of their strategic interdependence but even of the fact that their decisions are choices in a game of strategy.

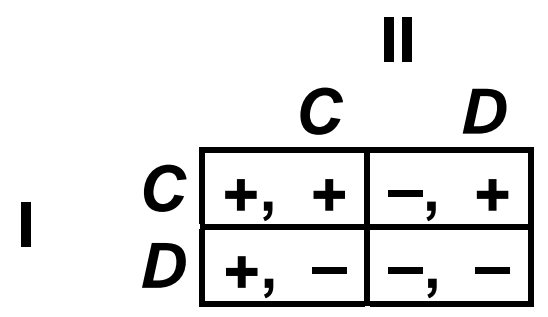

Figure 1. Mutual Fate Control game.

The payoff matrix of the game generally used to study the minimal social situation, named Mutual Fate Control by Thibaut and Kelley (1959, chapter 7), is displayed in Figure 1. Player I chooses between row $C$ and row $D$, Player II simultaneously (or, what amounts to the same thing, independently) chooses between columns $C$ and $D$, and the pair of symbols in each cell are the payoffs to Player I and Player II in that order. There is no need to attach numerical utilities to the payoffs; we assume merely that an outcome is either positive $(+)$ or negative (-) for each player. If both players choose the cooperative strategy $C$, then the outcome is shown in the upper-left cell, and both players receive positive payoffs. If both choose $D$, then both suffer negative payoffs. If one chooses $C$ and the other $D$, then the cooperator receives a negative payoff and the defector a positive payoff.

The Mutual Fate Control game can be interpreted as an impoverished Prisoner's Dilemma game, in which the level of measurement in the payoff functions is reduced to a binary scale (Sozański, 1992, p. 110). This is clarified in Figure 2. But the Prisoner's Dilemma game differs from the Mutual Fate Control game in important ways. In the Prisoner's Dilemma game, there is a unique (pure-strategy, strict) Nash equilibrium at $(D, D)$, representing joint defection. Furthermore, for each player, $D$ yields a strictly higher payoff than $C$ irrespective of the strategy chosen by the co-player, so that $D$ is a strongly dominant strategy. In the Mutual Fate Control game, on the other hand, all four outcomes $(C, C),(C, D),(D, C),(D, D)$ are (pure-strategy, weak) Nash equilibria, and there is no strongly dominant strategy. The characteristic feature of the Mutual Fate Control game, as its name suggests, is that the 
players' payoffs are unaffected by their own actions and are entirely in the hands of their coplayers - another feature not shared by the Prisoner's Dilemma game.

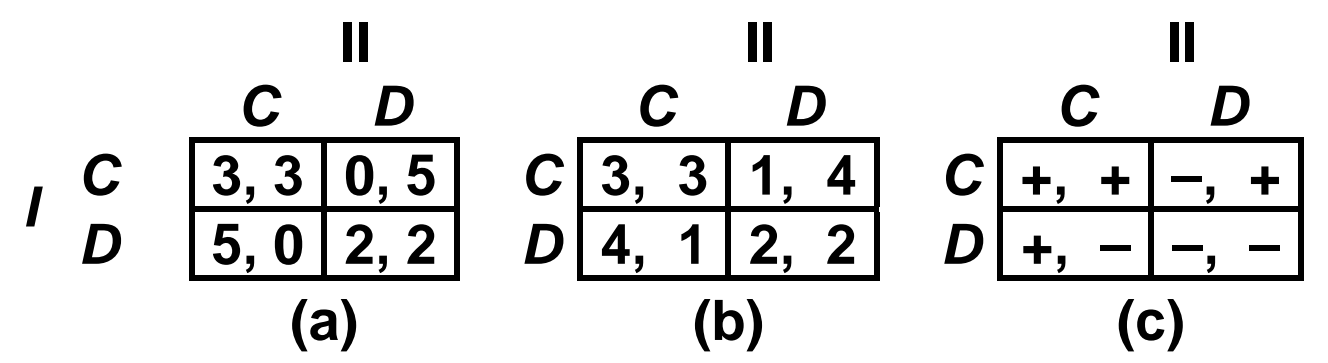

Figure 2. (a) Canonical Prisoner's Dilemma, with payoffs used by Axelrod (1994, 1997) and many other researchers. (b) Ordinal Prisoner's Dilemma, using ordinal payoffs 1, 2, 3, 4. (c) Mutual Fate Control, using binary payoffs.

\section{Intuitive background}

Could a Mutual fate Control game arise in a naturally occurring situation? Leaving aside the incomplete information that is characteristic of the MSS, there is no doubt that the Mutual Fate Control payoff structure is easily realized in everyday social interactions. Kelley and Thibaut (1978, pp. 5-13) discussed at some length an example taken from Tolstoy's novella, Family Happiness. Here is a simpler example of my own. A kidnapper has seized a wealthy industrialist's daughter and is threatening to kill her unless the industrialist pays a modest ransom. Let us assume that the kidnapper's strategy set consists of two actions: cooperate $(C)$ by sparing the hostage, or defect $(D)$ by killing her. The industrialist can cooperate by paying the ransom $(C)$, or defect by withholding it $(D)$. Assume also that the kidnapper prefers the ransom to be paid but is sufficiently ruthless to be indifferent as to whether the hostage is spared or killed, and that the industrialist prefers his daughter to be spared but is sufficiently wealthy or well-insured to be indifferent as to whether the ransom is paid or withheld. With these assumptions, the payoff structure evidently corresponds to the Mutual Fate Control game matrix shown in Figure 1.

The game may even arise in symbiotic relationships between non-human species. An obvious example from evolutionary biology is the symbiosis between a honeybee, which evolved (but might not have evolved) a strategy of transferring pollen from a flowering plant, and the plant, which evolved (but might not have evolved) a strategy of supplying nectar to the bee. If we make the admittedly strong simplifying assumption that the plant's marginal cost of producing nectar and the honeybee's marginal cost of carrying pollen are negligible in relation to the payoffs of the symbiosis, measured in units of Darwinian fitness (reproductive success), then this too is a Mutual Fate Control game.

It is more difficult to think of lifelike examples of the MSS in which the players are human and the necessary conditions of incomplete information are met. The following slightly artificial example of the cross-wired train is taken from Colman (1982a, pp. 289-91; 1995, pp. 40-50). ${ }^{6}$ Two people commute to work on the same train every weekday, always sitting in adjacent compartments. During the winter months, both compartments are uncomfortably cold. Each compartment has a lever marked 'heater', but there is nothing to indicate whether turning it to the left or to the right increases the temperature. (Up to this point, the story is hardly far-fetched - old British Rail rolling stock used to have heaters just like this.) Because of a fault in the electrical wiring of the train, moving either lever to the left increases the temperature, and moving it to the right decreases the temperature, in the adjacent compartment. The two commuters obviously have no direct control over the temperature in their own compartments. Their comfort is entirely in each other's hands, 
although neither of them knows this. But they would none the less both benefit if both turned their levers to the left at the beginning of every journey.

The following intriguing question arises: Can the commuters in the cross-wired train learn to cooperate by turning each other's heaters on in spite of being ignorant of their mutual dependence or even of each other's existence? More generally, can players learn to cooperate in a repeated MSS? If so, then cooperative behaviour, defined minimally as joint $C$-choosing, can evolve without conscious intention or awareness. Kelley (1968) named this phenomenon interpersonal accommodation, and if it is not itself a special form of teamwork, then it may at least help to understand some genuine forms of teamwork.

\section{Experimental findings}

The earliest MSS experiments (Sidowski, 1957; Sidowski, Wyckoff and Tabory, 1956) predated the rise of evolutionary game theory by three decades. They were based on a methodology and incentive scheme that seem slightly strange by contemporary standards. Pairs of players were seated in separate rooms, unaware of each other's existence, and electrodes were attached to their left hands. Each player was provided with a pair of buttons for choosing strategies and a digital display showing the cumulative number of points scored. The game was repeated many times, and on every round (repetition), each player pressed one of the buttons with the twin goals of earning a reward (point) and avoiding a punishment (painful electric shock). The experimental apparatus was arranged so that the rewards and punishments corresponded to the Mutual Fate Control payoff structure shown in Figure 1. Thus, pressing the left-hand button (labelled $C$ in Figure 1) caused the co-player to be rewarded with points, and pressing the right-hand button ( $D$ in Figure 1$)$ caused the co-player to be punished with electric shock (the functions of the left-hand and right-hand buttons were reversed for half the players). ${ }^{7}$

The findings showed that players generally learned to coordinate on the efficient $(C, C)$ Nash equilibrium, even when they were unaware of their strategic interdependence. After approximately two hundred repetitions, the relative frequency of $C$-choosing approached 7580 per cent. Sidowski (1957) ran some of his players in a non-MSS treatment condition in which they were informed that "there is another $S$ in another room who controls the number of shocks and the number of scores that you will receive. You in turn control the number of shocks and scores which the other $S$ receives' (p. 320). The results showed that this additional information did not lead to any increase in the relative frequency of cooperative choices $(\mathrm{p}$. 324). This suggests that whatever accounts for the evolution of cooperation in the MSS may also explain cooperation in more general strategic interactions calling for cooperation and teamwork. Under MSS conditions of incomplete information, players assumed that their payoffs were determined in some way by their own choices, but they tended to choose $C$ with increasing frequency over repetitions none the less. In the long run, some pairs of players settled down to choosing $C$ on every round. Players behaved as if they were learning to cooperate, although in the uninformed MSS treatment condition the situation was, from their point of view, non-interactive, and they did not know (and did not guess) that they had coplayers with whom to cooperate. This behaviour arguably represents the learning of a form of cooperation without awareness.

Following these early experiments, several further investigations of the MSS, using human and occasionally animal players, were published, with broadly similar findings (Bertilson and Lien, 1982; Bertilson, Wonderlich and Blum, 1983, 1984; Boren, 1966; Crawford and Sidowski, 1964; Delepoulle, Preux, and Darcheville, 2000; Kelley, Thibaut, Radloff and Mundy, 1962; Molm, 1981; Rabinowitz, Kelley and Rosenblatt, 1966; Sidowski and Smith, 1961). The structural properties of variations of the Mutual Fate Control game have been analysed mathematically and classified into equivalence classes and isomorphisms 
(Sozański, 1992), and computational studies have been undertaken of the performance of various stochastic learning models in the MSS (Arickx and Van Avermaet, 1981; Delepoulle, Preux and Darcheville, 2000).

How can the experimental findings be explained? Kelley, Thibaut, Radloff and Mundy (1962) proposed that players tend to adopt a myopic win-stay, lose-change (WSLC) strategy. This strategy is applicable to any repeated game based on a one-shot stage game with binary strategy sets - in which each player chooses from just two strategies on each round. Mutual Fate Control falls into this category. A player using the WSLC strategy repeats a stage-game strategy whenever it yields a positive payoff (above a specified aspiration level) and switches to the alternative stage-game strategy after receiving a negative payoff (below the aspiration level). In the Mutual Fate Control game, the aspiration level defines itself, because every payoff is obviously either positive $(+)$ or negative $(-)$; thus a player repeats the strategy chosen on the preceding round if it yielded a positive payoff and switches to the alternative strategy after receiving a negative payoff. Rapoport and Chammah (1965, pp. 73-4) rediscovered WSLC in a study of the repeated Prisoner's Dilemma game and called it Simpleton. Nowak and Sigmund (1993) rediscovered it again in their evolutionary research into repeated Prisoner's Dilemma games and called it Pavlov. ${ }^{8}$ Nothing quite like the Pavlov strategy was included in the computational studies mentioned at the end of the previous paragraph. The name Pavlov has caught on in the literature on evolutionary games, so I shall adopt it here. It turns out that this simple strategy leads to rapid evolution of cooperation in the MSS, as I shall now show.

\section{Formalization of dyadic MSS}

We can represent the outcomes on successive rounds of the MSS by a sequence of ordered pairs whose elements correspond to the choices of Player I and Player II respectively. For reasons of mathematical convenience that will emerge later, it will be useful to use the symbol 0 to represent $C$ and 1 to represent $D$. Let us assume that the game is repeated an indefinite number of times, and that the players' initial choices are arbitrary, but that on all subsequent rounds they use the Pavlov strategy. Assume first that both players initially choose 0 (cooperate). Because payoffs are positive, neither player will switch to the alternative strategy:

$$
(0,0) \rightarrow(0,0) \rightarrow(0,0) \rightarrow \ldots
$$

Next, assume that both players initially choose 1 (defect). In this case, both will receive negative payoffs, which will cause them to switch to 0 on the following round, after which they will repeat these 0 choices on all subsequent rounds, as already shown above. Thus:

$$
(1,1) \rightarrow(0,0) \rightarrow(0,0) \rightarrow \ldots
$$

Last, if one player initially chooses 0 and the other 1 , then the 0 -chooser will receive a negative payoff and will therefore switch to 1 on the following round, and the 1-chooser will receive a positive payoff and will therefore stay with 1 . On the second round, both players will therefore choose 1 , followed (as shown above) by 0 on all subsequent rounds:

$$
\begin{aligned}
& (0,1) \rightarrow(1,1) \rightarrow(0,0) \rightarrow(0,0) \rightarrow \ldots \\
& (1,0) \rightarrow(1,1) \rightarrow(0,0) \rightarrow(0,0) \rightarrow \ldots
\end{aligned}
$$

It is clear from this analysis that players who use the Pavlov strategy learn to cooperate - to choose mutually rewarding strategies - by the third round at the latest, and continue to 
cooperate indefinitely after that. Thus, if the commuters travelling on the cross-wired train discussed earlier were to adopt the Pavlov strategy, they would travel in comfort from the third journey onwards.

The Pavlov strategy is essentially a formalization of Thorndike's $(1898,1911)$ law of effect. Behaviour approximating it is observed widely in nature (Nowak and Sigmund, 1993), and it is the linchpin of Skinnerian learning theory; therefore it not only provides an explanation of the experimental findings but comes with a respectable theoretical and empirical pedigree in experimental psychology. Pavlov also has several properties that Axelrod $(1984,1997)$ identified as being characteristic of strategies that were most successful in his evolutionarily Prisoner's Dilemma tournaments. In particular, Pavlov is nice (never being the first to defect), forgiving (willing to cooperate at some point after the co-player has defected, if the co-player later cooperates, for example), and provocable (willing to retaliate in response to the co-player's defection). The experimental evidence is consistent with the hypothesis that human players implement the Pavlov strategy, or something resembling it, but not strictly (Burnstein, 1969; Crawford and Sidowski, 1964; Rabinowitz, Kelley and Rosenblatt, 1966; Sidowski, 1957; Sidowski, Wyckoff and Tabory, 1956; Sidowski and Smith, 1961). Cooperative choices usually begin to exceed chance frequency after a few rounds and continue to increase in frequency to about 75 per cent after 200 rounds. According to the Pavlov strategy, 100 per cent cooperation should occur after three rounds.

This suggests that if players use the Pavlov strategy, then they implement it imperfectly. None the less, in the light of evidence from the psychology of learning (e.g., Mackintosh, 1994), it seems highly likely that they are governed to a large extent by the law of effect, even if imperfectly. The formal analysis above is deterministic, but in other areas of evolutionary game theory, models often incorporate a stochastic element that has the effect of introducing noise into the process. The simplest method of introducing noise would be by assuming that there is a small probability $\varepsilon$ associated with each decision, causing a player to respond to a positive payoff by switching strategies, instead of repeating the same stage-game strategy, and to respond to a negative payoff by repeating strategies instead of switching. Introducing a stochastic element would not undermine the fundamental conclusion that the Pavlov strategy leads to the evolution of joint cooperation, but neither would it facilitate the adaptive process in this simple two-person model, a special form of randomness can facilitate cooperation in the multi-person model, as I shall show later. For the two-person case, Delepoulle, Preux and Darcheville (2000) showed that a more complex stochastic learning model developed by Staddon and Zhang (1991) yields results that most closely resemble the experimental data from human players in the MSS.

\section{Multi-person MMSS}

The multi-person minimal social situation (MMSS) is a generalization of the MSS to an arbitrary number of players (Coleman, Colman and Thomas, 1990). The set of $n \geq 2$ players, each with a uniquely designated predecessor and successor, may be represented by a cyclic graph of valency 2 . To visualize this structure, it is useful to imagine the $n$ players seated round a table. It is then intuitively obvious that Player l's predecessor is Player $n$ and that Player $n$ 's successor is Player 1. Each player has a choice of two strategies, 0 and 1 , hence the choices of the $n$ players on each repetition of the stage game can be represented by an $n$ vector of zeros and ones called a configuration. As in the two-person model discussed earlier, whenever a player chooses 0 , that player's successor receives a positive payoff, and whenever a player chooses 1 , that player's successor receives a negative payoff.

According to the Pavlov strategy, any player who receives a positive payoff will repeat the same strategy on the following round, and any player who receives a negative payoff will switch strategies on the following round. Every configuration therefore has a uniquely 
specified configuration that follows it according to the Pavlov strategy. It is obvious that a jointly cooperative configuration consisting entirely of zeros will be repeated on all subsequent rounds. Any configuration that leads ultimately to this zero configuration is called a cooperative configuration. The two-person MSS is a special case of the generalized MMSS, and the analysis of the MSS presented earlier shows that all configurations are cooperative in the dyadic case. However, I shall now show that is not true in general, by examining two illustrative configurations in the six-person MMSS (see Table 1).

\section{Table 1}

Configurations (strategy vectors) and payoff vectors in a repeated six-player MMSS starting from two different initial configurations

\begin{tabular}{llllllll}
\hline & Round 1 & Round 2 & Round 3 & Round 4 & Round 5 & Round 6 & Round 7 \\
\hline Configurations & $(1,0,1,0,1,0)$ & $(1,1,1,1,1,1)$ & $(0,0,0,0,0,0)$ & & & & \\
Payoff vectors & $(+,-,+,-,+,-)$ & $(-,-,-,-,-)$, & $(+,+,+,+,+)$, & & & & \\
& & & & & & & \\
Configurations & $(1,1,0,0,1,1)$ & $(0,0,1,0,1,0)$ & $(0,0,1,1,1,1)$ & $(1,0,1,0,0,0)$ & $(1,1,1,1,0,0)$ & $(1,0,0,0,1,0)$ & $(1,1,0,0,1,1)$ \\
Payoff vectors & $(-,-,-,+,+,-)$ & $(+,+,+,-,+,-)$ & $(-,+,+,-,-)$, & $(+,-,+,-,+)$, & $(+,-,-,-,-)$, & $(+,-,+,+,+,-)$ & $(-,-,-,+,+,-)$ \\
\hline
\end{tabular}

The configuration $(1,0,1,0,1,0)$ is followed by $(1,1,1,1,1,1)$, and then by $(0,0,0,0$, $0,0)$, which is repeated indefinitely. The initial configuration $(1,0,1,0,1,0)$ is therefore a cooperative configuration. The configuration $(1,1,0,0,1,1)$, on the other hand, generates the following sequence: $(1,1,0,0,1,1) \rightarrow(0,0,1,0,1,0) \rightarrow(0,0,1,1,1,1) \rightarrow(1,0,1,0$, $0,0) \rightarrow(1,1,1,1,0,0) \rightarrow(1,0,0,0,1,0) \rightarrow(1,1,0,0,1,1)$, returning to the starting point. This sequence will evidently cycle for ever, never reaching $(0,0,0,0,0,0)$. This shows that the initial configuration is not cooperative - and neither is any of the other configurations in the cycle.

An arbitrary configuration in an $n$-person MMSS can be represented by the vector

$$
\left(x_{1}, x_{2}, \ldots, x_{n}\right),
$$

where $x_{i} \in\{0,1\}$. It is helpful to consider 0 and 1 as elements of the Galois field GF(2) of integers modulo 2. A Galois field is a finite set of elements, together with operations of addition and multiplication satisfying axioms that govern addition and multiplication of rational numbers in conventional arithmetic. The binary Galois field GF(2), having just two elements labelled 0 and 1, and operations of addition and multiplication defined as in Figure 3, satisfies the axioms. GF(2) provides a convenient structure for modelling the MMSS, because it enables the transition from one configuration to the next under the Pavlov strategy to be expressed as a simple linear transformation.

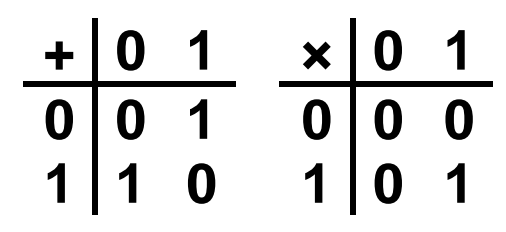

Figure 3. Addition and multiplication in the Galois field GF(2).

In the MMSS under Pavlov, the configuration $\left(y_{1}, \ldots, y_{n}\right)$ immediately following $\left(x_{1}, \ldots\right.$, $\left.x_{n}\right)$ is defined as follows: 


$$
y_{i}= \begin{cases}x_{i} & \text { if } x_{i-1}=0 \\ x_{i}+1 & \text { if } x_{i-1}=1\end{cases}
$$

where the subscripts are reduced modulo $n$. Since in $\operatorname{GF}(2) 0+0=1+1=0$, and $0+1=1+$ $0=1$,

$$
y_{i}=x_{i-1}+x_{i}(i=1, \ldots, n)
$$

The configuration immediately following $\left(x_{1}, \ldots, x_{n}\right)$ is therefore obtained by applying the linear transformation

$$
T:\left(x_{1}, \ldots, x_{n}\right)^{\prime} \rightarrow\left(x_{n}+x_{1}, x_{1}+x_{2}, \ldots, x_{n-1}+x_{n}\right)^{\prime}
$$

where $x^{\prime}$ denotes the transpose of the row vector $x$. The transformation matrix $T$ is shown below:

$$
T=\left(\begin{array}{llllll}
1 & 0 & 0 & 0 & \ldots & 1 \\
1 & 1 & 0 & 0 & \ldots & 0 \\
0 & 1 & 1 & 0 & \ldots & 0 \\
\vdots & & & & \\
0 & 0 & \ldots & 0 & 1 & 1
\end{array}\right)
$$

In this transformation matrix $T=\left[t_{i j}\right], t_{i j}=1$ if $i=j$ or $i=j+1(\bmod n)$, and $t_{i j}=0$ otherwise. If the initial configuration is $x=\left(x_{1}, \ldots, x_{n}\right)$, then the sequence of configurations (represented by transposed row vectors) on subsequent rounds will be $T x^{\prime}, T^{2} x^{\prime}, T^{3} x^{\prime}$, and so on. An initial configuration is cooperative if $x=\left(x_{1}, \ldots, x_{n}\right)$ lies in the kernel of the linear transformation $T^{k}$ for some $k$, that is, if for some $k, T^{k} x^{\prime}=(0,0, \ldots, 0)^{\prime}$.

What follows is a summary of some theorems regarding the MMSS under Pavlov, with proofs supplied in an appendix.

Theorem 1. If the configuration $\left(x_{1}, \ldots, x_{n}\right)$ is followed immediately by $(0,0, \ldots, 0)$, then either $\left(x_{1}, \ldots, x_{n}\right)=(0,0, \ldots, 0)$ or $\left(x_{1}, \ldots, x_{n}\right)=(1,1, \ldots, 1)$.

This theorem establishes that only configurations in which all players make the same choice as one another are immediately followed by joint cooperation. In any MMSS, joint cooperation is always preceded either by joint cooperation or by joint defection. This implies that unless the players all cooperate from the start, they must all defect on the same round before joint cooperation can occur.

Theorem 2. If $n$ is odd, then the only cooperative configurations are $(0,0, \ldots, 0)$ and $(1$, $1, \ldots, 1)$.

This implies that if the number of players is odd, then joint cooperation is achieved only if all players make the same initial choice, whether this is joint cooperation or joint defection, and it results after two rounds at most. The following two theorems are based on established properties of Galois fields. 
Theorem 3. If $j=2^{p}, p \in \mathbb{Z}^{+}$(where $\mathbb{Z}^{+}$is the set of non-negative integers) then $T^{j}$ : $\left(x_{1}\right.$, $\left.\ldots, x_{n}\right) \rightarrow\left(x_{1-j}+x_{1}, \ldots, x_{n-j}+x_{n}\right)$, where the subscripts are expressed modulo $n$.

This means that if $j$ is any number that is a power of 2 , then $j$ repetitions of the transformation $T$ take each component $x_{i}$ of the configuration $x$ into $x_{i-j}+x_{i}$. In other words, after 2 repetitions, the value of the component $x_{i}$ will be equal to $x_{i-2}+x_{i}$, bearing in mind that addition is modulo 2 , so that $0+0=1+1=0$, and $0+1=1+0=1$. Similarly, after 4 repetitions, $x_{i}$ will be equal to $x_{i-4}+x_{i}$, after 8 repetitions, $x_{i}$ will be equal to $x_{i-8}+x_{i}$, and so on for any power of 2 .

Theorem 4. A configuration $\left(x_{1}, \ldots, x_{n}\right)$ is a cooperative configuration iff $x_{i}=x_{i-k}$ for all $i$, where $n=b k, k=2^{a}, a, b \in \mathbb{Z}^{+}$(the set of non-negative integers), and $b$ is odd.

This theorem allows us to characterize the cooperative configurations of an $n$-person MMSS as follows. If $n$ is odd, then the cooperative configurations are $\left(x_{1}, \ldots, x_{n}\right)$ such that $x_{i}$ $=x_{i+1}$ for all $i(\bmod n)$. These are just the jointly cooperative and jointly defecting configurations $(0,0, \ldots, 0)$ and $(1,1, \ldots, 1)$ respectively. If $n$ is even, and if $k$ is the highest power of 2 that divides $n$ evenly, then the cooperative configurations are $\left(x_{1}, \ldots, x_{n}\right)$ such that $x_{i}=x_{i-k}$ for all $i(\bmod n)$. This means that, in an $n$-person MMSS, if $k$ is the highest power of 2 that divides $n$ evenly, then for the configuration to be cooperative, once the strategy choices of $k$ players have been specified, the choices of the remaining players are strictly determined. It follows that the number of cooperative configurations for the corresponding group size is $2^{k}$. In the two-person minimal situation, in which $k=2$, the number of cooperative configurations is 4 , confirming the analysis performed by enumeration for the dyadic case above. In larger MMSSs, however, cooperation evolves only in special cases.

Theorem 5. In a stochastic modification of the Pavlov strategy in which a player chooses 0 whenever a deterministic Pavlov player would choose 0, and chooses 1 with probability $p(0<p<1)$ whenever a deterministic Pavlov player would choose 1 with certainty, play converges in probability towards joint cooperation over repetitions in every MMSS.

The main conclusion of Theorem 4 was that, unless the number of players is a power of 2 , iterated play using the Pavlov strategy does not converge to joint cooperation except from special initial configurations. That conclusion was derived from a purely deterministic model in which players implement the Pavlov strategy mechanically. Here I consider a stochastic modification9 called Optimistic Pavlov in which a player who should, according to the deterministic Pavlov strategy, choose 1 (defect) with certainty defects with probability $p(0<$ $p<1$ ). Intuitively, this models a Pavlov-like player who prefers one of the strategies (0) to the other, and who follows the deterministic Pavlov strategy whenever it mandates a cooperative ( 0 ) choice, but chooses 1 with positive probability strictly less than unity when a deterministic Pavlov player would choose 1 with certainty. It is called Optimistic Pavlov because it mirrors the behaviour of a player with complete information who is generally cooperative and sanguine about the eventual evolution of joint cooperation and is somewhat reluctant to defect when deterministic Pavlov mandates defection. Theorem 5 establishes that Optimistic Pavlov play converges in probability towards joint cooperation in any MMSS, including an odd-sized MMSS.

\section{Predictions and conclusions}

The deterministic Pavlov theory yields predictions that are not obvious but are nevertheless empirically testable. First, although the relative frequencies of cooperative choices and joint 
cooperation tend to increase over repetitions in the two-person MSS, the theory predicts no such increases in odd-sized groups. Second, any even-sized MMSS in which the number of players is a power of two should behave like the two-person MSS: irrespective of the initial strategy choices, there should be progress toward joint cooperation when the game is repeated. Third, if the number of players is even but not a power of two, so that some initial configurations are cooperative according to the theory and others are not, then only the cooperative configurations should progress toward joint cooperation. Finally, in MMSSs of different sizes, proportions of cooperative choices and joint cooperative outcomes after many repetitions should correlate with the proportions of configurations that are cooperative according to the theory. Optimistic Pavlov theory, on the other hand, predicts that play will converge in probability towards joint cooperation in every MMSS.

It is difficult to perform the necessary experiments to test these predictions because of the large number of participants consumed in the course of experimental MMSS research. For statistical purposes, the unit of analysis has to be the group, because individual actions within groups are not independent, and this means that the number of participants required to study MSSSs increases rapidly with the number of players. This generates an associated problem of funding adequate incentives, which are considered necessary in contemporary experimental gaming. Furthermore, the players should ideally be isolated from one another and linked to an experimenter (but not to one another) by interactive computer terminals, which creates logistical problems. I have carried out some preliminary experiments, without monetary incentives or proper isolation. These were really only pilot studies with small numbers of groups. The results are interesting, however.

I found significantly smaller proportions of cooperative choices and joint cooperative outcomes in three groups playing the three-person MMSS than in three dyads playing the two-person MSS over 40 repetitions. Simple comparisons between the proportions of joint cooperative outcomes in MMSSs of different sizes can be misleading, because in the threeperson MMSS there is one joint cooperative configuration out of eight, whereas the twoperson MSS has one joint cooperative configuration out of only four, hence joint cooperation has a higher a priori probability of occurrence in smaller groups. However, cooperative individual choices increased markedly over four trial blocks of 10 rounds each in the twoperson MSS but not in the three-person MMSS, and that is in line with deterministic Pavlov theory.

I investigated three groups in the four-person MMSS over 40 repetitions. Very few joint cooperative outcomes occurred. The repetitions were divided into four trial blocks of 10 rounds each. In three of the groups, as predicted by both Pavlov and Optimistic Pavlov theory, significant increases in cooperative choices were observed, most of the increase appearing to occur between the first and second trial block. In one of the groups, the increase occurred only between the first and second trial block.

In groups of 2, 4, 8, 16, and so on, cooperation should invariably evolve. In groups of 6 , $10,12,14$, and so on, deterministic Pavlov theory predicts that cooperation should evolve from certain (identifiable) initial configurations and not from others, but these predictions are not necessarily robust in the presence of noise, because a single aberrant choice can take an MMSS out of a non-cooperative cycle. According to Optimistic Pavlov theory, cooperation should evolve in all these groups. The relevant data have yet to be collected. If any of the predictions are refuted by clear empirical evidence, then one or other of the theories will have to be rejected. In particular, the assumption that players approximate Pavlov or Optimistic Pavlov strategies will have to be abandoned.

The type of accommodative behaviour that evolves without awareness in the MMSS is arguably a form of cooperative teamwork meriting further attention. The introduction of a stochastic element into the Pavlov strategy seems to allow cooperation to evolve in groups of 
all sizes, provided that there is a reservoir of confidence causing players to be at least slightly reluctant to switch strategies after receiving negative payoffs. If this interpretation is correct, then cooperation should evolve without awareness in any group, among players who are more sanguine or tolerant than strict Pavlov players.

\section{Notes}

1. For a general discussion of interpersonal accommodation with and without awareness and communication in various types of interactions, including dyadic minimal social situations, see Kelley (1968).

2. For a simple proof, see Luce and Raiffa (1957, pp. 63-5), the most frequently cited version of von Neumann and Morgenstern's (1944, pp. 146-8) important result. The gist of the argument is that each player, knowing that the co-players are rational, expects them to choose strategies corresponding to the uniquely rational solution, and in turn chooses a utility-maximizing response to the co-players' strategies; hence every player chooses a utility-maximizing reply to the strategies of the co-players, yielding a Nash equilibrium by definition.

3. See von Neumann (1928, p. 295) and von Neumann and Morgenstern (1944, pp. 31-3). More than a decade after the appearance of von Neumann and Morgenstern's book, the authors of the leading textbook of game theory wrote: 'We feel that it is crucial that the social scientist recognize that game theory is not descriptive, but rather (conditionally) normative. It states neither how people do behave nor how they should behave in an absolute sense, but how they should behave if they wish to achieve certain ends' (Luce and Raiffa, 1957, p. 63, italics in original).

4. An evolutionarily stable strategy (ESS) is one with the property that if most members of a population adopt it, then no mutant strategy can invade the population by natural selection, and it is therefore the strategy that we should expect to see commonly in nature. Although an ESS is invariably a Nash equilibrium, the converse does not hold: a Nash equilibrium is not necessarily an ESS (Hofbauer and Sigmund, 1998, pp. 62-5; Weibull, 1995, pp. 48-50).

5. The preparation of this chapter was supported, in part, by Grant No. RES-000-23-0154 from the Economic and Social Research Council of the UK. I am grateful for helpful comments on an earlier version from participants at the Teamwork workshop and from my colleague Ali al-Nowaihi. This chapter draws heavily on the work reported in Coleman, Colman and Thomas (1990) and the discussion in Colman (1995, pp. 40-50).

6. For a completely different lifelike example, see Colman (1982b, pp. 37-44).

7. In later experiments with human players, the electric shocks were dispensed with, and the usual procedure was simply (and merely) to award points for positive payoffs and to deduct points for negative payoffs. Another peculiarity (and weakness) of the early experiments was that players made their choices as and when they wished, and the payoffs were delivered immediately.

8. The name Pavlov alludes to the strategy's reflex-like character. Nowak and Sigmund were unaware of the earlier publications of Kelley et al. and Rapoport and Chammah (Martin Nowak, personal communication). It is a striking indication of the gulf between game theorists and experimental psychologists that the name Pavlov continued to be used for many years in the literature of evolutionary games without anyone commenting that the strategy already had a name. It seems that the name win-stay, lose-change (WSLC) was not an evolutionarily stable meme, because Pavlov has largely supplanted it in the literature.

9. I am indebted to Ali al-Nowaihi for this interesting refinement. 


\section{References}

M. Arickx and E. Van Avermaet, 'Interdependent Learning in a Minimal Social Situation', Behavioral Science, 26 (1981) 229-42.

R. Axelrod, The Evolution of Cooperation. (New York: Basic Books, 1984).

R. Axelrod, The Complexity of Cooperation: Agent-based Models of Competition and Collaboration (Princeton, NJ: Princeton University Press, 1997).

H. S. Bertilson and S. K. Lien, 'Comparison of Reaction Time and Interpersonal Communication Tasks to Test Effectiveness of a Matching Strategy in Reducing Attackinstigated Aggression', Perceptual and Motor Skills, 55 (1982) 659-65.

H. S. Bertilson, S. A.Wonderlich and M. W. Blum, 'Withdrawal, Matching, Withdrawalmatching, and Variable-matching Strategies in Reducing Attack-instigated Aggression', Aggressive Behavior, 9 (1983) 1-11.

H. S. Bertilson, S. A.Wonderlich and M. W. Blum, 'Withdrawal and Matching Strategies in Reducing Attack-instigated Aggression', Psychological Reports, 55 (1984) 823-8.

J. J. Boren, 'An Experimental Social Relation Between Two Monkeys', Journal of the Experimental Analysis of Behavior, 9 (1966) 691-700.

E. Burnstein, 'The role of reward and punishment in the development of behavioral interdependence', in J. Mills (Ed.), Experimental Social psychology (London: Macmillan, 1969), pp. 341-405.

A. A. Coleman, A. M. Colman and R. M. Thomas, 'Cooperation Without Awareness: A Multiperson Generalization of the Minimal Social Situation', Behavioral Science, 35 (1990) $115-21$.

A. M. Colman (ed.), Cooperation and Competition in Humans and Animals (Wokingham: Van Nostrand Reinhold, 1982a).

A. M. Colman, Game Theory and Experimental Games: The Study of Strategic Interaction (Oxford: Pergamon, 1982b).

A. M. Colman, Game Theory and Its Applications in the Social and Biological Sciences (2nd edn, London: Routledge, 1995).

T. Crawford and J. B. Sidowski, 'Monetary Incentive and Cooperation/Competition Instructions in a Minimal Social Situation', Psychological reports, 15 (1964) 233-4.

S. Delepoulle, P. Preux and J.-C. Darcheville, 'Evolution of Cooperation Within a Behaviorbased Perspective: Confronting Nature and Animats', Artificial Evolution Lecture Notes in Computer Science, 18291 (2000) 204-16.

J. Hofbauer and K.Sigmund, Evolutionary Games and Population Dynamics (Cambridge: Cambridge University Press, 1998). 
H. H. Kelley, 'Interpersonal accomodation', American Psychologist, 23 (1968) 399-410.

H. H. Kelley and J. W. Thibaut, Interpersonal Relations: A Theory of Interdependence (New York: Wiley, 1978).

H. H. Kelley, J. W. Thibaut, R. Radloff and D. Mundy, The Development of Cooperation in the "Minimal Social Situation", Psychological Monographs, 76 (1962) Whole No. 19.

R. D. Luce and H. Raiffa, Games and Decisions: Introduction and Critical Survey (New York: Wiley, 1957).

N. J. Mackintosh (Ed.), Animal Learning and Cognition: Handbook of Perception and Cognition (2nd edn, San Diego, CA: Academic Press, 1994).

L. D. Molm, 'A Contingency Change Analysis of the Disruption and Recovery of Social Exchange and Cooperation', Social Forces, 59 (1981) 729-51.

J. F. Nash, 'Non-cooperative Games', in H. W. Kuhn and S. Nasar, Eds, The Essential John Nash (Princeton, NJ: Princeton University Press, 2002) pp. 51-84.

M. A. Nowak and K. Sigmund, A Strategy of Win-stay, Lose-shift that Outperforms Tit-forTat in the Prisoner's Dilemma Game', Nature, 364 (1993) 56-8.

L. Rabinowitz, H. H. Kelley and R. M. Rosenblatt, 'Effects of Different Types of Interdependence and Response Conditions in the Minimal Social Situation', Journal of Experimental Social Psychology, 2 (1966) 169-97.

A. Rapoport and A. M. Chammah, Prisoner's Dilemma: A Study in Conflict and Cooperation (Ann Arbor, MI: University of Michigan Press, 1965).

J. B. Sidowski, 'Reward and Punishment in the Minimal Social Situation', Journal of Experimental Psychology, 54 (1957) 318-26.

J. B. Sidowski and M. Smith, 'Sex and Game Instruction Variables in a Minimal Social Situation', Psychological Reports, 8 (1961) 393-7.

J. B. Sidowski, L. B. Wyckoff and L. Tabory, 'The Influence of Reinforcement and Punishment in a Minimal Social Situation', Journal of Abnormal and Social Psychology, 52 (1956) 115-19.

T. Sozański, 'A Combinatorial Theory of Minimal Social Situations', Journal of Mathematical Sociology, 17 (1992) 105-25.

J. E. R. Staddon and Zhang, Y., 'On the assignment-of-credit problem in operant learning', in M. L. Commons, S. Grossberg and J. E. R. Staddon, Eds, Neural Network Model of Conditioning and Action: The XIIth Harvard Symposium (Hillsdale, NJ: Erlbaum, 1991) pp. 279-93.

J. W. Thibaut and H. H. Kelley, The Social Psychology of Groups (New York: Wiley, 1959). 
E. L. Thorndike, 'Animal Intelligence: An Experimental Study of the Associative Processes in Animals', Psychological Review Monograph Supplement, 2(4) (1898) Whole No. 8.

R. L. Thorndike, Animal Intelligence: Experimental Studies (New York: Macmillan, 1911).

J. von Neumann, 'Zur Theorie der Gesellschaftsspiele', Mathematische Annalen, 100 (1928) 295-320.

J. von Neumann and O. Morgenstern, Theory of Games and Economic Behavior. (Princeton, NJ: Princeton University Press, 1944).

J. W. Weibull, Evolutionary Game Theory (Cambridge, MA: MIT Press, 1995).

\section{Appendix}

Proofs of Theorems 1 to 4 were given in Coleman, Colman and Thomas (1990) and are reproduced here for convenience.

Theorem 1. If the configuration $\left(x_{1}, \ldots, x_{n}\right)$ is followed immediately by $(0,0, \ldots, 0)$, then either $\left(x_{1}, \ldots, x_{n}\right)=(0,0, \ldots, 0)$ or $\left(x_{1}, \ldots, x_{n}\right)=(1,1, \ldots, 1)$.

Proof. If $x_{i}=0$, then $x_{i-1}=0$, otherwise the $i$ th component of the transformed vector $T x^{\prime}$ would be 1 . Similarly, bearing in mind that $1+1=0$ in $\mathrm{GF}(2)$, if $x_{i}=1$, then $x_{i-1}=1$, otherwise the $i$ th component of the transformed vector would be 1 .

Theorem 2. If $n$ is odd, then the only cooperative configurations are $(0,0, \ldots, 0)$ and $(1$, $1, \ldots, 1)$.

Proof. If $T x^{\prime}=(0,0, \ldots, 0)^{\prime}$, then it follows from Theorem 1 that either $x=(0,0, \ldots, 0)$ or $x=(1,1, \ldots, 1)$, and if $(0,0, \ldots, 0)$ is not the initial configuration, then it must be preceded by $(1,1, \ldots, 1)$. Suppose that $(1,1, \ldots, 1)$ is also not the initial configuration. Then $T w^{\prime}=(1,1, \ldots$, $1)^{\prime}$ for some $w$. Now if $w_{i}=0$, then $w_{i-1}=1$, otherwise the $i$ th component of $T w^{\prime}$ would be zero. For the same reason, if $w_{i}=1$, then $w_{i-1}=0$. Therefore, $w_{i-2}=w_{i}$. Consider the vector component $w_{n}$. Because $n$ is odd, $w_{n}=w_{n-2}=\ldots=w_{1}$. This implies that if $w_{1}=0$, then $w_{1-1}=$ $w_{n}=0$, and that if $w_{1}=1$, then $w_{1-1}=w_{n}=1$, which yields a contradiction.

Theorem 3. If $j=2^{p}, p \in \mathbb{Z}^{+}$(where $\mathbb{Z}^{+}$is the set of non-negative integers) then $T^{j}$ : $\left(x_{1}\right.$, $\left.\ldots, x_{n}\right) \rightarrow\left(x_{1-j}+x_{1}, \ldots, x_{n-j}+x_{n}\right)$, where the subscripts are expressed modulo $n$.

Proof. Assume that the result is true for some $p$. Then, if $q=2^{p}, T^{q}:\left(x_{1}, \ldots, x_{n}\right) \rightarrow\left(x_{1-q}+x_{1}\right.$, $\left.\ldots, x_{n-q}+x_{n}\right)$. The proof proceeds by induction on $p$. For $p+1,2^{p+1}=2 q$, and $T^{2 q}=T^{q} T^{q}$. Now

$$
T^{2 q}=T^{q} T^{q}:\left(x_{1}, \ldots, x_{n}\right) \rightarrow\left(y_{1}, \ldots, y_{n}\right),
$$

where $y_{i}=\left(x_{i-2 q}+x_{i-q}\right)+\left(x_{i-q}+x_{i}\right)=x_{i-2 q}+x_{i}$, because, whether $x_{i-q}=0$ or $1, x_{i-q}+x_{i-q}=0$. Thus,

$$
T^{2 q}:\left(x_{1}, \ldots, x_{n}\right) \rightarrow\left(x_{1-2 q}+x_{1}, \ldots, x_{n-2 q}+x_{n}\right)
$$


We have proved that if the result holds for same $p$ then it holds for $p+1$. The final step is to show that it holds for $p=0$. In that case, $q=2^{0}=1$, and $T^{1}=T$ is the basic transformation

$$
T:\left(x_{1}, \ldots, x_{n}\right) \rightarrow\left(x_{n}+x_{1}, x_{1}+x_{2}, \ldots, x_{n-1}+x_{n}\right),
$$

for which the result holds. We have therefore proved that if $j$ is any number that is a power of 2 , then $j$ repetitions of the transformation $T$ takes each component $x_{i}$ of the configuration $x$ into $x_{i-j}+x_{i}$.

Theorem 4. A configuration $\left(x_{1}, \ldots, x_{n}\right)$ is a cooperative configuration iff $x_{i}=x_{i-k}$ for all $i$, where $n=b k, k=2^{a}, a, b \in \mathbb{Z}^{+}$(the set of non-negative integers), and $b$ is odd.

Proof. Theorem 3 established that

$$
T:\left(x_{1}, \ldots, x_{n}\right) \rightarrow\left(x_{1-j}+x_{1}, \ldots, x_{n-j}+x_{n}\right),
$$

where $j=2^{p}, p \in \mathbb{Z}^{+}$. Hence the kernel of $T^{j}$ is

$$
\operatorname{ker} T^{j}=\left\{\left(x_{1}, \ldots, x_{n}\right) \mid x_{1-j}+x_{1}=\ldots=x_{n-j}+x_{n}=0\right\}=\left\{\left(x_{1}, \ldots, x_{n}\right) \mid x_{i}=x_{i+j} \text { for all } i\right\} .
$$

Because $\operatorname{ker} T^{p}$ is a subset of $\operatorname{ker} T^{p+1}$ for all $p \in \mathbb{N}$ (the set of natural numbers), the set of cooperative configurations is $\operatorname{ker} T^{p}$ if $k e r T^{p}=\operatorname{ker} T^{m}$ for $p<m$. A constructive proof of this begins by establishing that if $k=2^{a}, m=2 k=2^{a+1}$, then $\operatorname{ker} T^{k}=\operatorname{ker} T^{m}=\operatorname{ker} T^{2 k}$.

Let $c=(b+1) / 2$. Then $b=2 c-1$, and $k b=k(2 c-1)$. Hence $2 c k \equiv k(\bmod k b)$, that is,

$$
c m \equiv k(\bmod n) .
$$

It now follows that if $x \in \operatorname{ker} T^{m}$, then $x_{i}=x_{i+m}$ for all $i(\bmod n)$, and hence that $x_{i+m}=x_{i+2 m}=$ $x_{i+3 m}=\ldots$, and hence, because $c \in \mathbb{Z}^{+}$,

$$
x_{i}=x_{i+c m} \text { for all } i(\bmod n) \text {. }
$$

Because $c m \equiv k(\bmod n)$

$$
x_{i}=x_{i+k} \text { for all } i(\bmod n) \text {, }
$$

and this shows that $x \in \operatorname{ker} T^{k}$, and hence that $\operatorname{ker} T^{m}$ is a subset of $\operatorname{ker} T^{k}$. Furthermore, because $k<m$,

$$
\operatorname{ker} T^{k}=\operatorname{ker} T^{m}
$$

This completes the proof.

Theorem 5. In a stochastic modification of the Pavlov strategy in which a player chooses 0 whenever a deterministic Pavlov player would choose 0 , and chooses 1 with probability $p(0<p<1)$ whenever a deterministic Pavlov player would choose 1 with certainty, play converges in probability towards joint cooperation over repetitions in every MMSS. 
Proof. If Player $i-1$ chooses $x_{i-1}$ and Player $i$ chooses $x_{i}$ in Round 0, then in Round 1 the probability $p\left(x_{i}^{1}=1\right)$ that Player $i$ defects (chooses 1$)$ is $p\left(x_{i}^{1}=1\right)=p\left(x_{i-1}^{0}+x_{i}^{0}\right)$, and in general

$$
p\left(x_{i}^{k}=1\right)=p\left(x_{i-1}^{k-1}+x_{i}^{k-1}\right)(i=1, \ldots, n),
$$

where $x_{i}^{k}$ is the strategy choice of Player $i$ in Round $k$, addition is $\bmod 2$ (with $1+1=0$ ), and subscripts are reduced $\bmod n$ (with $i-i=0=n$ ). Because $x_{i-1}^{k-1}+x_{i}^{k-1} \in\{0,1\}$, it follows that $p\left(x_{i}^{k}=1\right) \in\{0, p\}$, and $p\left(x_{i}^{k}=1\right)=p$ iff $x_{i-1}^{k-1}+x_{i}^{k-1}=1$. If $p\left(x_{i}^{k}=1\right)=p$, then, on subsequent rounds, $p\left(x_{i}^{k+1}=1\right)=p^{2}, p\left(x_{i}^{k+2}=1\right)=p^{3}$, and so on. Furthermore, $0<p<1$, hence $p^{r}$ tends to zero as $r$ increases. Therefore, $p\left(x_{i}^{k}=1\right)=p^{r}$ unless on any round $k-1$ the value of $x_{i-1}^{k-1}$ changes, in which case $p\left(x_{i}^{k}=1\right)=0$. It follows that, over $r$ repetitions of the MMSS, $p\left(x_{i}^{r}=1\right)$ tends to zero for all $x_{i}$ and therefore that the outcome configuration converges in probability towards joint cooperation. This completes the proof. 